\title{
Parts with Internal Programmed Geometry Produced Using Additive Manufacturing
}

\author{
Części z programowaną geometrią wewnętrzną \\ wytwarzane przyrostowo - części PGW
}

MACIEJ CADER *

Article presents results of rapid strength estimation method and optimization algorithm for internal geometry of parts produced using additive manufacturing. Proposed new naming associate with rapid prototyping - parts with internal programmed geometry - IPG Part.

KEYWORDS: 3D printing, strength estimation, internal geometry selection, parts production

The dynamic development of incremental technologies (additive / 3D printing) has made them an increasingly important alternative to standard manufacturing technologies. Incremental production consists of the assembly of subsequent layers of material, based on a 3D CAD model, such as machine parts. This part can have almost any external and internal geometry. Because of the way they are built layer by layer - these technologies give the possibility of so-called. Programming the internal geometry (fig. 1). This means that the internal geometry of the machine parts can be optimized (programmed) for external load, operating conditions or other factors (e.g. minimizing the total weight of the part with its original external shape).

a)

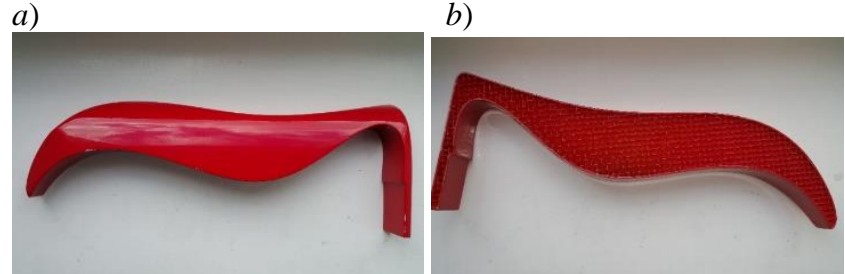

Fig. 1. Example of a polymeric part produced by incremental method with programmable internal geometry: a) varnished outer surface, b) internal geometry (mesh) adjusted to boundary conditions

Due to the programmable internal geometry, machine parts can be optimized so that they meet the required functions at the prescribed strength. Going forward - the mass of "passive" machines, ie the mass of the covers, bodies and housings, can be reduced to the required minimum with the required strength. Knowing the design and implementation of such components will translate into improvements in machine performance - for example, weight reduction will allow for smaller / cheaper powertrains. In addition, it is possible to build ultra-light machine bodies whose response to an external factor (e.g. impact) is fully predictable thanks to the programmed internal geometry.

\footnotetext{
* Dr inż. Maciej Cader (mcader@piap.pl) - Przemysłowy Instytut Automatyki i Pomiarów PIAP
}

DOI: https://doi.org/10.17814/mechanik.2017.7.94

Despite the dynamic development of incremental production technology (3D printing), the development of software or methods that give the opportunity to consciously exploit the potential of these technologies is slow. This is evidenced by a world-wide review in this area and literature.

There are no advanced work on algorithms for combining three important aspects of optimizing machine parts by incremental methods. This is where the geometry of the fill is made, on this basis - the final shape and distribution of the interior geometry (CAD 3D visualization) is generated, and on this basis, the tool path for the manufacturing head is proposed. The elements of these aspects of optimum production though appearing in different degrees in the world - are in no way intertwined. Going forward, it is not yet possible to transfer even the results of topological analysis of the shape - done on the basis of the finite element method - directly to the practice. On the other hand, the dynamic development of additive manufacturing machines that meet industry standards and requirements (3D printers) allows the production of virtually any shape from a wide range of materials (polymers, alloys, precious metals, ceramics, etc.). However, the machine capabilities are not optimally utilized, precisely because of the difficulty of translating the results of MES analysis into machine language, which would allow the generation of a tool path based on the specific geometry of the produced part.

\section{Method of selecting the geometry of the inner part}

The development of a method for selecting the internal geometry of incremental prototypes is based on the FDM technology, which gives the greatest possibilities in this respect. FDM consists of applying further layers of thermosetting polymer (e.g. ABS-M30) in the form of a thin fiber of isotropic material. However, a prototype with a pre-programmed filling-internal geometry, as a whole, exhibits mechanical properties such as orthotropic material. This is due to the capabilities and characteristics of FDM technology:

- arbitrary control of the material filament in the horizontal plane,

- building models layer by layer.

This has been proven. in own research [1] and in other centers [e.g. 2-4]. Therefore, the model of orthotropic material should be used. In geometry of this type one can distinguish three directions perpendicular to one another, called the principal material axes (fig. 2). 


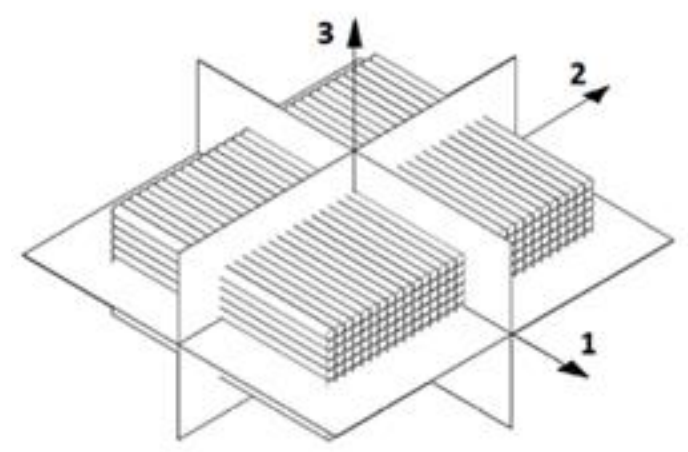

Fig. 2. Sketch of incremental geometric structure in FDM technology: 1 and 2 - direction of fiber laying, 3 - direction of geometry increment along vertical axis

Since the FDM geometry has an orthotropic property, according to the generalized Hooke law, constitutive equations that determine the mechanical properties of this structure can be written in the form of the following matrix equation:

$$
\left\{\begin{array}{l}
\varepsilon_{11} \\
\varepsilon_{22} \\
\varepsilon_{33} \\
\gamma_{23} \\
\gamma_{31} \\
\gamma_{12}
\end{array}\right\}=\left[\begin{array}{cccccc}
\frac{1}{E_{11}} & -\frac{v_{21}}{E_{22}} & -\frac{v_{31}}{E_{33}} & 0 & 0 & 0 \\
-\frac{v_{12}}{E_{11}} & \frac{1}{E_{22}} & -\frac{v_{32}}{E_{33}} & 0 & 0 & 0 \\
-\frac{v_{13}}{E_{11}} & -\frac{\nu_{23}}{E_{22}} & \frac{1}{E_{33}} & 0 & 0 & 0 \\
0 & 0 & 0 & \frac{1}{G_{23}} & 0 & 0 \\
0 & 0 & 0 & 0 & \frac{1}{G_{31}} & 0 \\
0 & 0 & 0 & 0 & 0 & \frac{1}{G_{12}}
\end{array}\right]\left\{\begin{array}{c}
\sigma_{11} \\
\sigma_{22} \\
\sigma_{33} \\
\tau_{23} \\
\tau_{31} \\
\tau_{12}
\end{array}\right\}
$$

where: $\sigma_{i j}$ and $T_{i j}$ - stress vector components, $\varepsilon_{i j}$ and $\gamma_{i j}$ the components of the deformation vector ( $i$ and $j$ may assume values 1,2 or 3 ) and the rigidity matrix is a symmetric matrix of 12 independent magnitudes:

- three Young modules: $E_{11}, E_{22}, E_{33}$,

- three Kirchhoff modules: $G_{23}, G_{31}, G_{12}$,

- and six Poisson modules: $v_{21}, v_{31}, v_{12}, v_{32}, v_{13}, v_{23}$ where for FDM: $v_{12}=v_{21}, v_{13}=v_{31}, v_{23}=v_{32}$.

After adopting the orthotropic assumption of the geometric structure produced incrementally in FDM and obtaining valuable values (elastic data) on the basis of experimental studies, computer properties of the geometric structure can be calculated [5].

The effectiveness of the method was analyzed in the uniaxial stretching of normative samples and field deflection tests of FDM-produced prototype parts that contained representative internal fill geometries (fig. 3).
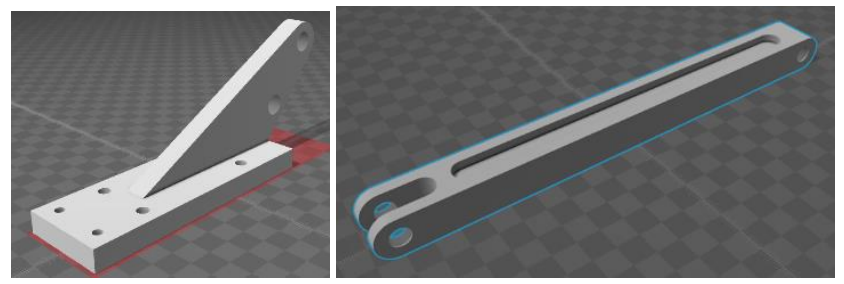

Fig. 3. Tested prototypes produced in three batches containing different geometries of internal filling: solid-fiber, ribbed and grid [5]
Based on the studies [5], elastic constants for the geometry of the discussed geometries and strength characteristics were determined. Additionally, field deflection of prototype parts with these internal geometries was investigated. The spring constants have been used for simulated one-axis stretching of normative samples and simulated prototype deflection studies designed in normal modeling mode as solid CAD bodies. The results of laboratory and simulation studies were compared. In addition, the results were compiled with the results of tests of samples and prototypes made by injection molding of material ABS-M30, i.e. with an isotropic structure. Information about the accuracy of the strength calculations was obtained. It has been shown [5] that in the case of the estimation of strength on the basis of simulated tests performed for the CAD geometry exhibiting isotropic properties, the error was in the range of $30.8-87.0 \%$. For the same prototypes, the strength estimation error using the proposed method was in the range of $12.2-39.8 \%$. The error value depends on the type of geometry of the prototype fill. This means that the error in estimation of strength using the proposed method has decreased on average by half compared to the previous simulation method based on the solidisotropic geometry of the prototypes. Fig. $4 \mathrm{a}$ and b show four graphs:

- error curve between computer simulation results for the developed MSS method (based on the obtained results) and laboratory results,

$\longrightarrow$ - error curve between computer simulation results for isotropic structures and laboratory results.

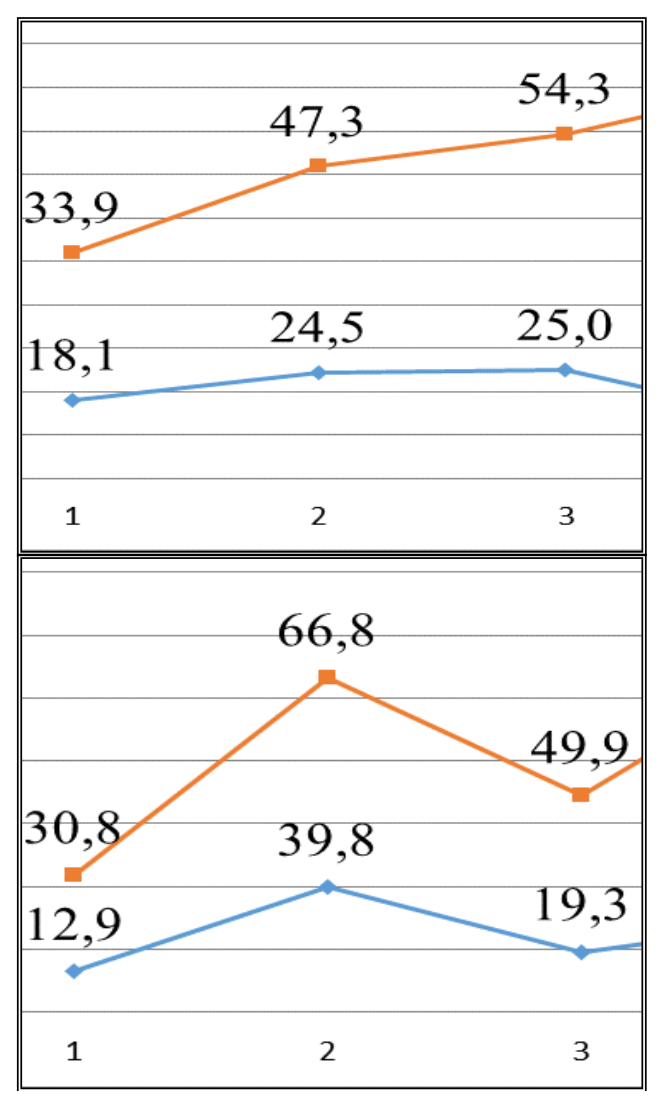

Fig. 4. The curves of error values in \%, calculation of deflection of: a) the arm b) the jaw - for three geometries of filling: 1 - solid (fiber), 2 - ribbed 3 - grid 


\section{Optimization algorithm for geometry selection}

In parallel with the work on the selection of internal geometries for incremental prototypes in the Rapid Prototyping and Numerical Methods Laboratory, PIAP began work on the creation of software that allows the selection of internal geometries for incremental parts. The selection algorithm will be based on functional assumptions of topological optimization performed using MES and user-defined boundary conditions. In addition, the program will use the algorithm to determine the path of a tool selected for FDM add-on technology, ie, taking into account its features. It was assumed that the user would receive a $3 \mathrm{D}$ geometry that would be the basis for programming the corresponding machine movements direct production on a PIAP 3D printer (fig. 5).

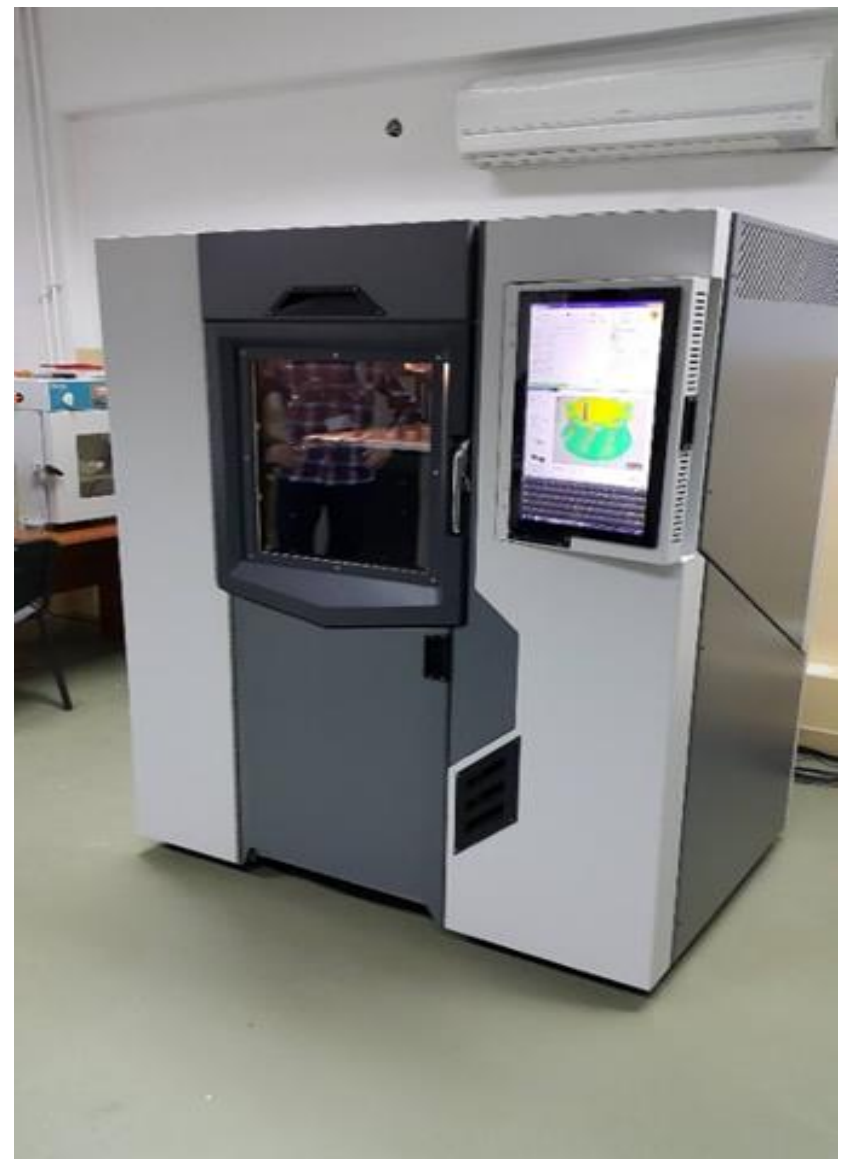

Fig. 5. 3D printer produced by PIAP

Briefly, the principle of operation of the program is as follows: the user will be able to import into the environment of the $3 \mathrm{D}$ geometry program of the part to be created in additive technology. The software will change the graphic representation of the body to a skeleton. Skeletal representation of the solid will be applied to the grid of points, but in a way that is correlated with the principle of building models in FDM. This means that the mesh density (e.g. the cuboidal points and the connecting lines) will correspond to the thickness of the applied layers and to how the machines work. It was assumed that the density of the net would be adjustable but within the specified range due to the limitations of additive technology. The grid will be cut off by the skeleton model, and points will be created at the intersection of the grid and the solid surface to be joined by the corresponding lines. This will create a volumetric and surface grid for the model. Using the surface net formed on the surface, the user will be able to indicate the areas of occurrence of the load. The loads will be in the form of force or pressure. The software defines using the developed mathematical models - the area of influence of the applied load field on the grid nodes. Areas - based on the algorithm - will be determined by four categories: strong, moderate, weak, no impact. This user will be able to learn from the visualization. In the next step, the software proposes to select and distribute the density of the internal geometry and will create tool paths based on curves stretched at the points of the grid. The user will be able to make corrections to the proposed path and its final shape. It was assumed that in the last phase of the program, the user would be able to export the model file to a file containing the prepared geometry as a surface solid - STL or for applications on the PIAP additive machine - to start the manufacturing process.

\section{Conclusions}

The author proposes to introduce to the nomenclature related to fast prototyping techniques and technologies a new concept: "parts with internal geometry" - IPG Parts.

In the Laboratory of Rapid Prototyping and Numerical Calculations PIAP, works on three aspects related to parts of PGW intended for production using additive methods (3D printing):

- developing methods for rapid modeling of any geometry shapes of internal parts of PGW parts,

- developing methods of rapid estimation of the strength of PGW parts in order to optimize the additive process,

- optimization of production methods by selecting the internal geometry of the PGW part.

\section{REFERENCES}

1.Cader M., Jastrzębska A., Pakieła Z., Szwejkowski P. „Analiza wpływu orientacji modelu w komorze roboczej na wytrzymałość w procesie FDM". Mechanik. 7 (2012): pages 85-94.

2.Arivazhagan A., Masood S.H. „Dynamic response of FDM made ABS parts in different part orientations". AdvMaterRes. 748 (2013): pages 291-294.

3.Croccolo D., De Agostinis M., Olmi G. „Experimental characterization and analytical modelling of the mechanical behaviour of fused deposition processed parts made of ABSM30". Computational Materials Science. 79 (2013): pages 506-518.

4.Domingo-Espin M., Puigoriol-Forcada J. „Mechanical property characterization and simulation of fused deposition modeling Polycarbonate parts". Materials \& Design. 83 (2015): pages 670-677.

5.Cader M. „Szacowanie wytrzymałości prototypów wytwarzanych przyrostowo metodą FDM”. Warszawa: Oficyna Wydawnicza PIAP, 2016. 